\title{
Learned Multi-Patch Similarity
}

\author{
Wilfried Hartmann ${ }^{1} \quad$ Silvano Galliani $^{1} \quad$ Michal Havlena $^{2}$ \\ Luc Van Gool ${ }^{3,4} \quad$ Konrad Schindler ${ }^{1}$ \\ ${ }^{1}$ Photogrammetry and Remote Sensing, ETH Zurich, Switzerland \\ ${ }^{2}$ Vuforia, PTC, Vienna, Austria \\ ${ }^{3} \mathrm{CVL}$, ETH Zurich, Switzerland $\quad{ }^{4}$ PSI, KU Leuven, Belgium
}

\begin{abstract}
Estimating a depth map from multiple views of a scene is a fundamental task in computer vision. As soon as more than two viewpoints are available, one faces the very basic question how to measure similarity across $>2 \mathrm{im}$ age patches. Surprisingly, no direct solution exists, instead it is common to fall back to more or less robust averaging of two-view similarities. Encouraged by the success of machine learning, and in particular convolutional neural networks, we propose to learn a matching function which directly maps multiple image patches to a scalar similarity score. Experiments on several multi-view datasets demonstrate that this approach has advantages over methods based on pairwise patch similarity.
\end{abstract}

\section{Introduction}

3D reconstruction from two or more images of the same scene is a central problem in computer vision. Assuming that the camera poses are already known, the problem reduces to (multi-view) stereo matching, i.e., establishing dense point correspondences between the images, which can then be converted to 3D points by triangulating the corresponding rays. The core of stereo matching itself is a function to measure the similarity between points in different images, respectively between the points surrounding image patches. Once such a similarity measure is available, it can be computed for a list of different putative correspondences to find the one with the highest similarity.

For the classic two-view stereo case, the definition of a similarity function is comparatively straight-forward: transform the image intensities of the two patches such that more similar ones end up closer to each other, according to some pre-defined distance metric. Many methods have been proposed, including simple sum-of-squared differences, (inverse) normalized cross-correlation to afford

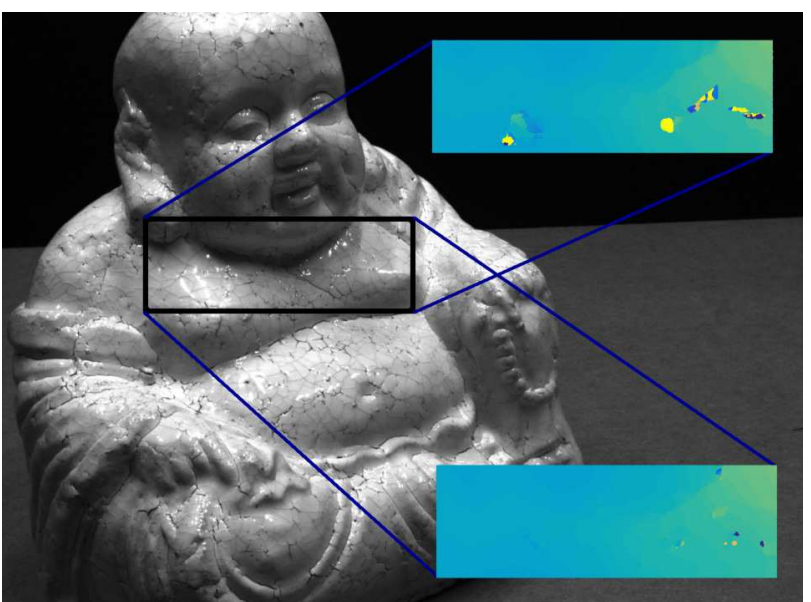

Figure 1. Multi-view depth estimation. A conventional, pairwise similarity like ZNCC (top) is unable to find the correct depth in corrupted regions, e.g. specular reflections; whereas the proposed multi-view similarity (bottom) can predict correct depth values.

invariance against linear brightness changes, and even more robust measures like the Hamming distance between Census descriptors. More recently it has also been proposed to learn the distance metric discriminatively from matching and non-matching training examples.

In practice, multi-view stereo is often the method of choice, since the higher redundancy and larger number of viewpoints allows for more robust $3 \mathrm{D}$ reconstruction. A host of rather successful multi-view stereo methods exist (see benchmark results such as [12, 18, 19, 22]). Surprisingly, these methods in fact have no mechanism to measure the similarity between $>2$ image patches that form a putative multi-view correspondence. Instead, they heuristically form a consensus over the pair-wise similarities, or a subset of them (most often the similarities from all other stereo partners to a "reference image" in which the depth map is 
parametrized). We note that the same is true for "multi-view stereo" methods that reconstruct implicit [25] or explicit [5] surfaces. These either reconstruct points from two images and delay the multi-view integration to the surface fitting stage; or they measure photo-consistency between pairs of images, or between some "mean" surface texture and the individual images.

Here, we pose the question why not directly measure multi-view similarity? Encouraged by the successes of learned similarity measures, we propose a multi-stream ("Siamese") convolutional neural network architecture that takes as input a number of $n>2$ image patches, and outputs a scalar similarity score. The network is learned directly from matching and non-matching example patches, keeping decisions like the right weighting of individual images or image pairs (e.g., to account for varying contrast) or the robustness of the consensus mechanism (e.g., due to occlusions, specularities, and other disturbances in individual images) implicit. An alternative view of our work is as a multi-view extension of learning-based stereo correspondence [23, 26, 27] to more than two views. We posit that the learning-based stereo should profit from the multi-view setup, precisely because the additional images afford the necessary redundancy to detect and resolve situations where two-view stereo struggles. To test our similarity measure, we embed it into a standard depth estimation setup, namely multi-view plane-sweeping [4]: for each pixel in an image we compute similarity scores for a range of depths along the ray, and pick the depth with the highest similarity.

There are different strategies to cast stereo matching into a machine learning problem. One can either fix the metric (e.g., Euclidean distance) and learn to map image patches to "descriptor vectors" that, according to that metric, have small distance for matching patches and large distance for non-matching patches [23]. For our case, that approach does not resolve the problem of defining an $n$-view distance. Alternatively, one can map raw patches to descriptors according to some conventional recipe, e.g. SIFT, and train a similarity/dissimilarity metric between them [24]. However, given the spectacular improvements that learned features from CNNs have brought to computer vision, we prefer to learn the mapping end-to-end from raw pixel intensities to a similarity score.

Conceptually, it is straight-forward to design a CNN for multi-view similarity. A standard architecture to jointly process two images with similar image statistics are "Siamese" networks: the two inputs are passed through identical streams with tied weights and then combined for the final decision layers by simple addition or concatenation. We do the same for $n>2$ images and set up $n$ parallel streams with tied weights, without introducing additional free parameters. The network is trained on a portion of the public DTU multi-view dataset [12], and evaluated on the remain- ing part of it, as well as on an unrelated public dataset. We will also show that it is possible to vary the number $n$ of input image patches at test time without retraining the network. The comparison to other conventional and learningbased approaches demonstrates the benefit of evaluating direct multi-view similarity, especially in the case when the reference view is corrupted, e.g., due to specular reflection - see Figure 1

\section{Related Work}

Depth Estimation. An enormous body of literature exists on stereo matching. Much of the early work addressed the minimal two-view case [1]. Given the relative pose of the two images, stereo correspondence reduces to a 1D search along corresponding epipolar lines. It is standard practice to impose a smoothness prior, both along and across epipolar lines, to better cope with ambiguities of the local similarity measure. For disparity map computation a commonly used measure is the sum of absolute differences (SAD) [14]. Here the similarity measure is based on intensity differences of two image patches with the same size. Zero-mean normalized cross correlation (ZNCC) [11] is another popular similarity measure, which features invariance against linear brightness changes. A discussion of the complete twoview stereo literature is beyond the scope of this paper, for an overview and further reading please refer to benchmarks like the Middlebury Stereo page [17] or KITTI [8].

While not as over-whelming as in the two-view case, there still is an extensive literature about multi-view stereo. Conceptually, there are two main approaches to generate depth maps from multiple views. One exhaustively tests for all possible depths, often by "sweeping" a fronto-parallel plane through the 3D object space along the depth axis [4] or in multiple directions [10] to sample different depth values efficiently. The other avoids exhaustive testing and instead relies on efficient random sampling and propagation schemes like PatchMatch [2] to find a good depth estimate at every position, e.g. [6]. For both strategies, efficient GPU implementations exist. Again, we refer the reader to benchmark datasets such as [19] and [12] for an overview of contemporary multi-view stereo algorithms. We note that there is also a large body of work termed "multi-view reconstruction" that in fact computes depth maps from two views and focuses on integrating the corresponding 3D point clouds into implicit [7, 25] or explicit [5] surface representations. Since these methods only start after having lifted image points to 3D scene space, they are not directly relevant for the present paper.

Patch Similarity Learning. With the rise of machine learning for computer vision problems, it has also been proposed to learn the similarity measure for (two-view) stereo. Early work still relied on hand-tuned descriptors such as 
SIFT, the learning served to "distort" the descriptor space so that nearby false matches get pushed apart and the distance becomes more discriminative [24]. The advent of deep learning suggested that the bottleneck might be the descriptors themselves rather than the distance metric, so it was proposed to learn similarity directly from raw images [27]. Closely related work started from separate steps for descriptor learning and metric learning, and unified them to effectively obtain a direct similarity prediction from raw image data [9] as well. An extensive study of similarity measures based on different CNN architectures is presented in [26]. That work also showed that CNN-based similarities outperform both classical descriptor spaces like SIFT [16] and other learned descriptors such as [21]. Another strategy is to learn patch descriptors, but freeze the distance measure used to compare them. The recently proposed LIFT descriptor [20, 23] is learned with the help of a Siamese network, using a loss function that ensures that descriptors of matching patches end up having low Euclidean distance, whereas non-matching descriptors have not. The learned output is a 128-dimensional descriptor vector which corresponds to the size of the SIFT descriptor vector [16] so that LIFT can serve as a drop-in replacement for SIFT or similar handcoded descriptors in existing matching pipelines.

Yet, the learned descriptors still share the limitation of most two-view stereo methods, that similarity is measured only for image pairs, as a distance in descriptor space.

\section{Measuring $n$-way Patch Similarity with a Neural Network}

We aim for a function that directly maps $n>2$ image patches $p_{i}$ to a scalar similarity score $S\left(p_{1}, p_{2}, \ldots, p_{n}\right)$. Our proposed solution is to learn that function from example data, in the spirit of what is sometimes called "metric learning" 1 As learning engine, we use a convolutional neural network. We point out that the strategy to learn a multipatch similarity is generic, and not limited to stereo correspondence. In fact, a main argument for learning it is that a learned score can be tuned to different applications, just by choosing appropriate training data. In our target application, the $n$ patches are the reprojections of a candidate $3 \mathrm{D}$ point into $n$ different views of the scene.

For our purposes, we make the assumption that every set of patches in the training data is either "similar" or "dissimilar", and do not assign different degrees of similarity. I.e., we cast the similarity as a binary classification problem. Conveniently, this means that the similarity score is bounded between 0 (maximally dissimilar) and 1 (maximally similar). Such a hard, discriminative approach reflects the situation of stereo matching (and correspondence

\footnotetext{
${ }^{1}$ We refrain from using that name, since the learned similarity score is not guaranteed to be a metric in the mathematical sense.
}

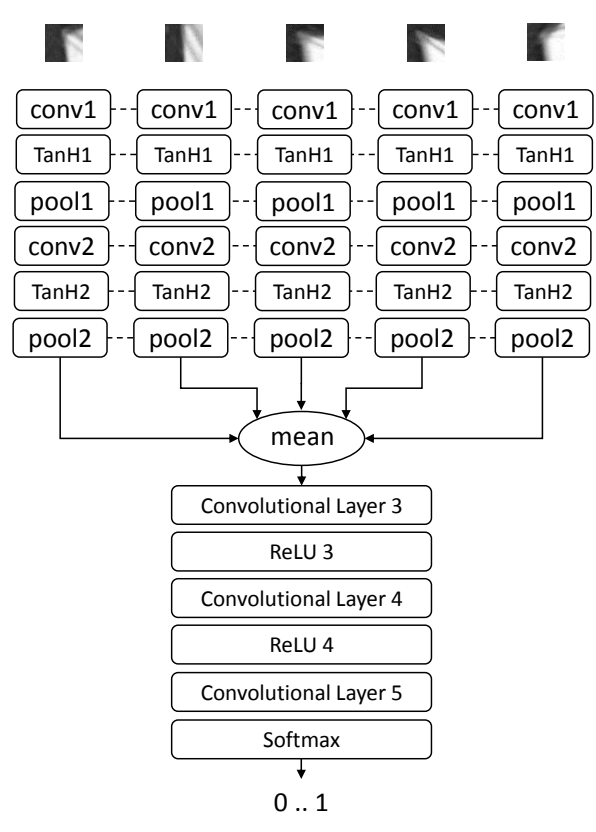

Figure 2. The proposed network architecture with five "Siamese" branches. The input are the five patches, the output is the similarity score that is used to select the correct depth value

estimation in general), where for any given pixel in a reference image there is only one correct answer, namely the set of patches that correspond to the ground truth depth. We note that for other applications, for example image retrieval, the definition may not be suitable and would have to be replaced with a more gradual, continuous one (for which it is however not as straight-forward to generate training labels).

\subsection{Network Architecture}

The network we use for learning a depth map is illustrated in Figure 2, Its inputs are $n$ image patches (w.l.o.g. we set $n=5$ for the remainder of the paper) of size $32 \times 32$ pixels. The early layers process each patch separately with the same set of weights, corresponding to an $n$-way Siamese network architecture. Identical weights in the different branches are a natural choice, since the result should not depend on the order of the input patches. Note that the number of free weights to be learned is independent of the number $n$ of views.

Each branch starts with a convolutional layer with 32 filter kernels of size $5 \times 5$. Then follow a tanh non-linearity and a max-pooling layer with kernel size $2 \times 2$. That same sequence is then repeated, this time with 64 filter kernels of size $5 \times 5$, and $\max$-pooling over $2 \times 2$ neurons. From the resulting $5 \times 5 \times 64$ layers the mean is taken over all $n$ branches. The output is passed through two convolutional layers with 2048 filter kernels, each followed by a $R e L U$ non-linearity, and a final convolutional layer with 2 filter kernels. The final network output is the similarity score. 
The described, fully convolutional architecture has the advantage that it is much more efficient to compute for larger images at test time than a per-pixel similarity. Due to the two pooling layers, the network outputs a similarity score for every $4 \times 4$ pixel region.

When designing the network, the straightforward approach would be to concatenate the outputs of the individual branches, instead of averaging them. We evaluate both options and find their performance on par, see Tab. 2 Note that averaging the branch outputs makes it possible to input a varying number of views without retraining the network.

As usual, the exact network design is found empirically and is somewhat hard to interpret. We tested several different architectures and found the described one to work best. Compared to other current architectures for image analysis, our network needs fewer convolutional layers, presumably because of the small input patch size; and, interestingly, oldschool tanh non-linearities work better than $R e L U$ in the early layers.

\section{Application to Multi-view Stereo}

Having introduced the multi-view similarity computation, we use it as a building block in a fairly standard multiview stereo pipeline [10], assuming known camera poses (e.g., from prior structure-from-motion computation). As usual, one camera is designated as the reference view for which the depth map is computed. We emphasize that, other than in most existing multi-view approaches, the reference view serves only to fix the parametrization of the depth map. In our method, its patches do not have a privileged role as the "source" templates to be combined to the "target" patches of the stereo partners in a pairwise fashion. Note, in a multi-view setup it can happen that points are occluded in the reference view, but visible in several other views. In that case the learned similarity score may assign the highest similarity to a point that is invisible in the reference image. If the final product of multi-view stereo is a 3D point cloud, this behavior does not hurt (except that the corresponding point on the occluder is missing).

To find the most likely depth for a given pixel, we discretize the depth along the viewing direction of the reference view, as in standard plane-sweep stereo. Matching then reduces to exhaustively testing all possible depth values and choosing the one with the highest similarity. For a given patch in the reference view the matching patches in the other images are extracted. This is repeated for all planes, where each plane corresponds to a discrete depth value. Each $n$-tuple of patches (including the reference patch, which is the same for every depth) is fed to the similarity network. Not surprisingly, rather larger patches give more reliable similarity estimates, but there is a trade-off against memory consumption and computational cost. We found $32 \times 32$ pixels to be a good compromise.

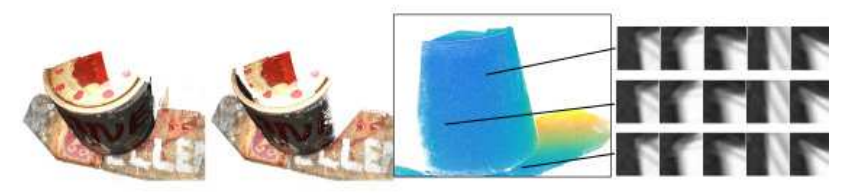

Figure 3. Generating training data. The ground truth 3D point cloud is processed using the visibility check of [15]. Points not visible in the reference view are removed. Next, points are randomly selected, projected into the image, and a patch centered at the projection is cropped out.

Training the Network. Our network is implemented in Caffe [13], and learned from scratch. As training data, we sample image patches from 49 scenes of the DTU dataset [12] (Fig. 3).

Positive samples (similarity 1) are selected using the ground truth depth information. Negative samples are selected by picking patches from depth planes 15 steps before or after the ground truth. Note the power of the learning approach to optimally tune to the application: patches on correct epipolar lines are the only ones the network will ever see at test time (assuming correct camera poses). Using only such patches for training ensures that the model is "complete", in the sense that it sees all sorts of patch combinations that can occur along epipolar lines; but at the same time it is also "tight" in the sense that it does not contain irrelevant negatives that do not correspond to any valid set of epipolar geometries and merely blur the discriminative power of the similarity.

It is a recurring question what proportion of positive and negative samples to use in situations with very unbalanced class distributions (for multi-view stereo, only one sample within the entire depth range is positive for each pixel). For our case it worked best to use a balanced training set in each training batch, which also speeds up convergence. In total, we sample 14.7 million positive and 14.7 million negative examples. Learning is done by minimizing the softmax loss w.r.t. the true labels with 500,000 iterations of standard Stochastic Gradient Descent (SGD), with batch size 1024; starting from a base learning rate of 0.001 , that is reduced by a factor of 10 every 100,000 iterations.

\section{Experiments and Results}

To evaluate our learned similarity measure, we conduct multi-view depth estimation experiments on several scenes. In the first experiment, we demonstrate the performance of our approach using the DTU [12] evaluation framework and compare it to four existing baseline methods. Second, we test the similarity network's ability to generalize across different environments. Without retraining the network, we feed it with input images having different lighting condi- 
tions, depth range, and sensor characteristics than what was observed during training.

The way the similarity measure is employed to provide depth map predictions is the same for all the compared approaches. After picking a reference view, we find the four camera viewpoints closest to it. These five images and their camera poses are fed to a plane-sweeping routine we use the publicly available code of [10]. The explored depth range is set according to the given dataset, and warped views are generated for 256 uniformly sampled disparity levels. The SAD and ZNCC similarity measures, already implemented in the plane-sweeping library, are used to select the best depth estimate for each pixel position in the reference view, based on the $4 \times 256$ pairwise similarity scores. To ensure a fair comparison with the proposed approach, the same patch size of $32 \times 32$ pixels is used.

For the other compared descriptors, SIFT [16] and LIFT [20, 23], we compute similarity via the pairwise (Euclidean) descriptor distances from the warped images, and feed them to the same decision mechanism to select the best per-pixel depth estimate. These descriptors were designed to work with patch size $64 \times 64$ pixels, so we use these dimensions, even though it gives them the advantage that they can base their prediction on $4 \times$ more pixels. Note, the warping already corrects for scale and orientation, so we skip the corresponding parts and directly compute the descriptor entries from the warped images. We point out that this comparison is not completely fair, since the two descriptors were designed for sparse interest-point matching, where some translational invariance is desirable. Still, we wanted to test against LIFT, as the most recent learned, CNN-based descriptor. For completeness, we include its hand-crafted counterpart.

In order to have a computationally efficient depth map prediction with the proposed approach, we choose the input patch size to be $128 \times 128$ pixels. This allows the network to compute the similarity scores for $25 \times 25$ partially overlapping patches (stride 4 ) of size $32 \times 32$ in a single forward pass, filling up a region of $100 \times 100$ similarity scores (after upsampling). Doing this for every depth of the sweeping plane, we obtain a list of 256 multi-view similarity scores per pixel, and simply pick the depth with the highest score. To compute the $25 \times 25 \times 256$ similarity scores (covering an area of $100 \times 100$ original pixels) takes $70 \mathrm{~ms}$, on an Intel i7 computer with Nvidia Titan X GPU using Caffe in Linux.

Practical matching software does not return raw depth estimates, but improves them with different simple steps. The plane-sweeping library [10] offers two such options: (i) interpolation of the discrete depth levels to sub-pixel accuracy, and (ii) box filtering to account for correlations between nearby depth values. We tested both options and found them to consistently improve the overall results, independent of the similarity measure. As we are primarily interested how well different similarity metrics perform under realistic conditions, we enable subpixel accuracy and box filtering in all following experiments.

\subsection{Evaluation on the DTU dataset}

The DTU Robot Image Data (DTU) contains more than 80 different indoor table top scenes. Each DTU scene was recorded from predetermined, known camera poses with the help of a camera mounted on a robot arm, and a ground truth 3D point cloud was generated by mounting a highaccuracy active stereo sensor on the same robot arm. Images from 49 scenes are already used as our training data. Beyond those, also scenes that share one or more objects with any of the training scenes are excluded from testing, to avoid potential biases. For DTU, we set the depth range to $[0.45 \ldots 1] \mathrm{m}$.

Qualitative Results. The four example objects we use in the following are labeled BIRD, FLOWER, CAN, and BUDDHA. The BIRD has a uniform color so the intensity differences are largely due to the shading effects. The FLOWER is a difficult object due to the thin structure and the small leaves. Underneath the flowerpot, there is a newspaper with strong texture. The CAN has a metallic surface with homogeneous texture, while the background is rather strongly textured. The BUDDHA is made out of porcelain, with significant specular reflections.

The provided color images are converted to grayscale before processing, $c . f$. Fig. 4a $4 \mathrm{~d}$ The ground truth depth maps have been generated by back-projecting ground truth 3D point clouds and are sparse, c.f. Fig. 4e $4 \mathrm{~h}$, whereas the depth maps from multi-view stereo are dense. We thus evaluate only at the sparser ground truth depths. Also the difference plots in Fig. 4i $4 \mathrm{t}$ show only the pixels for which ground truth is available. Depth differences are color-coded on a logarithmic scale, where dark blue corresponds to zero difference and dark red denotes differences $>20 \mathrm{~mm}$.

For the BIRD the right wing, which is partly in shadow, is the most difficult part. For all three methods there are errors at the wing boundary, the largest errors are observed for LIFT. Note also errors on the left wing boundary present in ZNCC and LIFT but not in the proposed method. The most difficult object is the FLOWER. Here the ZNCC and our approach again outperform LIFT. All three methods struggle with the dark, homogeneous soil in the flowerpot. On the leaves, as far as present in the ground truth, our method has the smallest error, especially near the boundaries. For the CAN object, the homogeneous metal surface challenges the LIFT approach, whereas the two others can resolve it correctly. For the BUDDHA the most difficult parts for depth estimation are the small regions with specular reflection. These can be seen in Figs. 1, 4d In those regions ZNCC and LIFT have large errors, while our direct multi-view ap- 


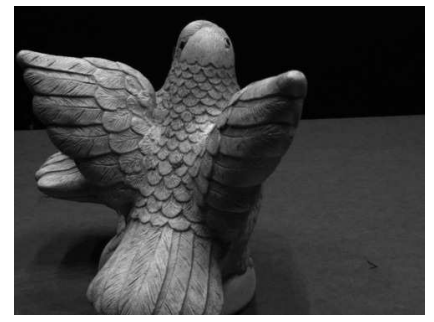

(a)

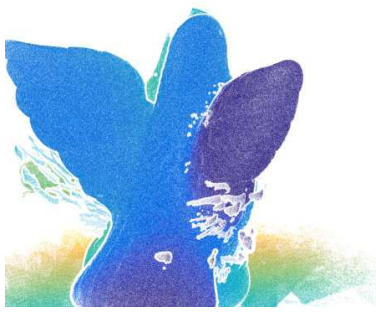

(e)

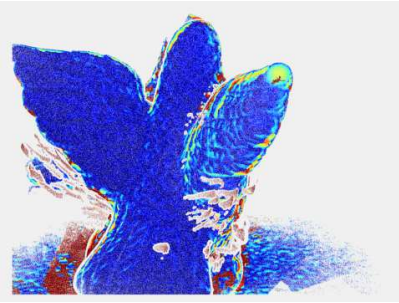

(i)

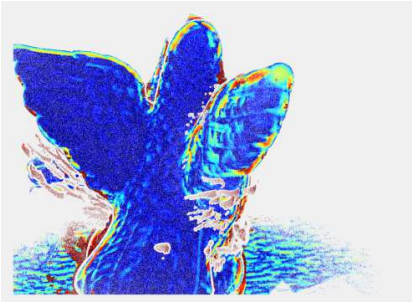

(m)

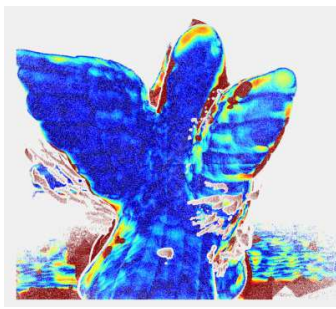

(q)

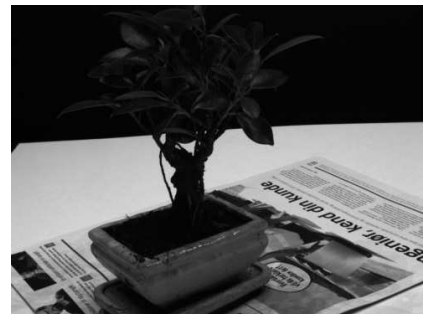

(b)

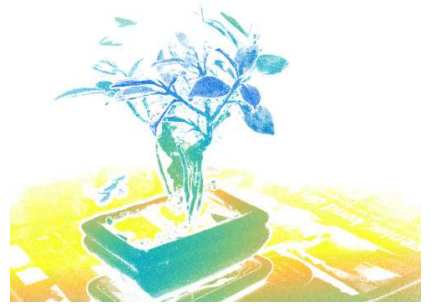

(f)

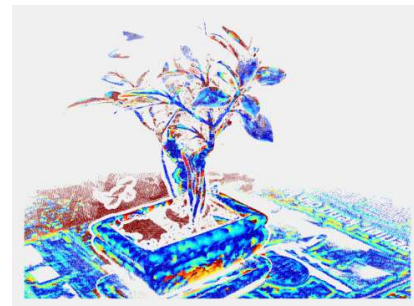

(j)

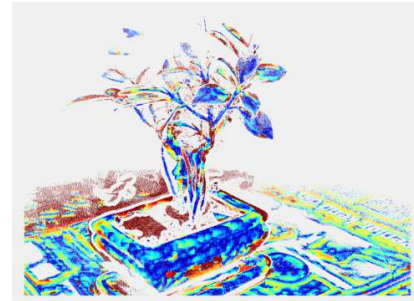

(n)

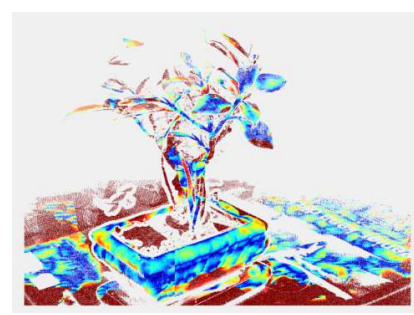

(r)

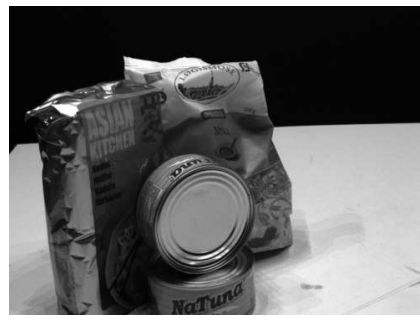

(c)

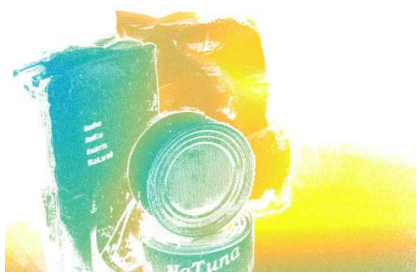

(g)

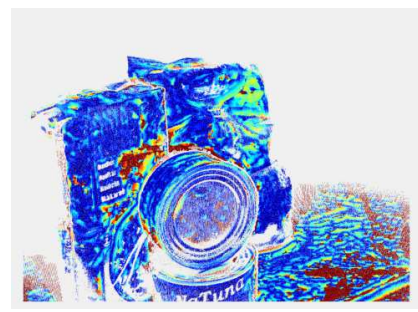

(k)

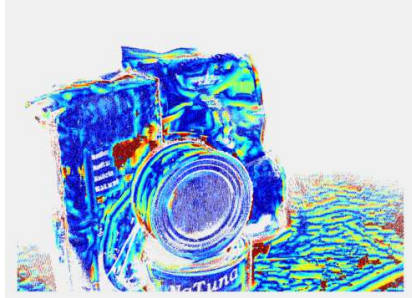

(o)

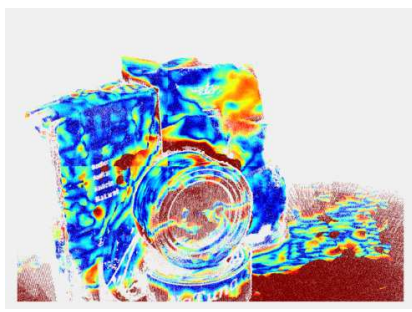

(s)

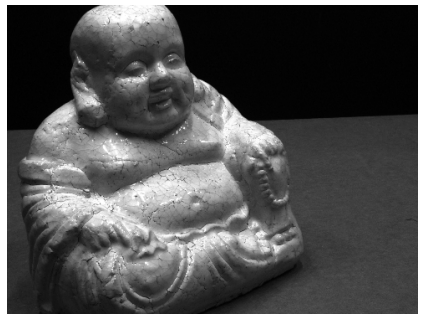

(d)

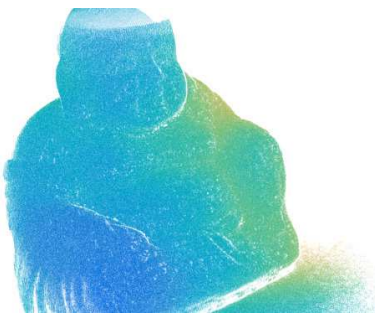

(h)

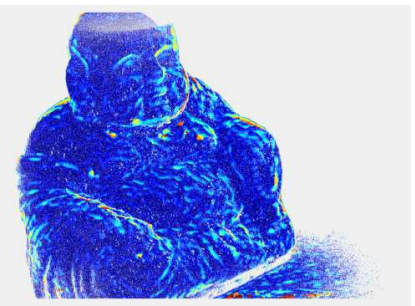

(1)

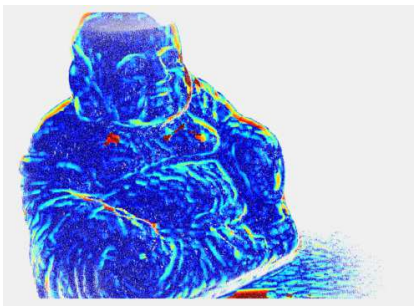

(p)

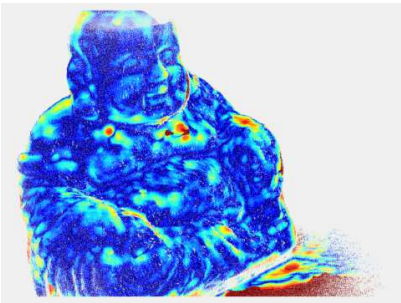

(t)

Figure 4. Plane sweeping using three different patch similarity measures. Proposed learned multi-view similarity $v s$. pairwise ZNCC and pairwise LIFT. Reference images (a-d). Ground truth (e-h). Absolute deviations from ground truth for the proposed method (i-k), ZNCC $(\mathrm{m}-\mathrm{p})$ and LIFT (q-t). Blue: no difference, red: high difference.

proach copes a lot better (Fig. 41). Overall, the examples suggest that our proposed similarity is more general: the classic ZNCC method often works well, but struggles near object boundaries. LIFT seems to have problems with cer- tain surfaces materials like the metal of the CAN, and both competitors struggle with specularities (which, incidentally, are a main problem of stereo reconstruction, especially in industrial applications). 


\begin{tabular}{|l|c|cc|cc|}
\hline Similarity & \#views & \multicolumn{2}{|c|}{ Accuracy } & \multicolumn{2}{c|}{ Completeness } \\
& & Mean & Median & Mean & Median \\
\hline SAD & $4 \times 2$ & 1.868 & 0.617 & 2.534 & 1.148 \\
ZNCC & $4 \times 2$ & $\mathbf{1 . 2 3 7}$ & 0.577 & 2.260 & 1.025 \\
OUR stereo & $4 \times 2$ & 1.339 & $\mathbf{0 . 4 5 3}$ & 2.964 & 1.391 \\
OUR 5-view & 5 & 1.356 & 0.472 & $\mathbf{2 . 1 2 6}$ & $\mathbf{0 . 8 6 8}$ \\
\hline
\end{tabular}

Table 1. Quantitative results for 20 objects from the DTU datasets. Four similarity measures are compared: sum of absolute differences, zero-mean normalized cross correlation, proposed similarity over 2 views, and proposed similarity over 5 views.

Quantitative results. The authors of the DTU dataset provide an evaluation framework in which, per object, the groundtruth 3D point cloud is compared to the one constructed from the depth estimates. For the evaluation we thus convert the estimated depth maps to $3 \mathrm{D}$ point clouds by lifting them with the known camera calibration. Note that we do not use depthmap integration across multiple viewpoints. Accuracy is defined as the average (truncated) distance from a reconstructed point to the nearest ground truth point. Completeness is defined inversely, as average distance from ground truth points to the nearest model points, i.e., lower values are also better.

We start with a comparison to standard similarity measures for 20 scenes in Table 1 . While the mean values describe the overall performance, median values are less sensitive to outliers. Note that, also for the mean, the per-point distances of outliers are truncated at $20 \mathrm{~mm}$. To support the claim that direct multi-view similarity is preferable to consensus over 2-view scores, we also run our method in 2 -view stereo mode and feed the scores to the same consensus mechanism used for SAD and ZNCC. Directly computing similarity over 5 input views delivers significantly better completeness at similar accuracy as the best competitors. We did not run the full experiment with LIFT, due to the excessive runtime of pixelwise $\mathrm{CNN}$ prediction without a fully convolutional architecture.

In Table 2 we compare the accuracy and completeness of all tested methods for the four example scenes. Differences are relatively small between SAD and ZNCC, probably due to the controlled lighting. The results for SIFT and LIFT are also quite similar, except for the CAN object where SIFT clearly outperforms its learned counterpart.

The proposed method achieves the best median accuracy and median completeness in all the scenes, and the best mean accuracy and completeness for three of them. Note that there is virtually no difference between averaging and concatenation. There seems to be no performance penalty for averaging, while at the same time one gains the flexibility to use a variable number of input views. On the BIRD, our method ranks third in accuracy and second in completeness. There are relatively big differences between median

\begin{tabular}{|l|ll|ll|}
\hline Similarity & \multicolumn{2}{|c|}{ Accuracy } & \multicolumn{2}{c|}{ Completeness } \\
(5 views) & Mean & Median & Mean & Median \\
\hline \multicolumn{5}{|c|}{ BIRD } \\
\hline SAD & 2.452 & 0.380 & $\mathbf{4 . 0 3 5}$ & 1.105 \\
ZNCC & $\mathbf{1 . 3 7 5}$ & 0.365 & 4.253 & 1.332 \\
SIFT & 1.594 & 0.415 & 5.269 & 1.845 \\
LIFT & 1.844 & 0.562 & 4.387 & 1.410 \\
OUR concat & 1.605 & 0.305 & 4.358 & 1.133 \\
OUR & 1.881 & $\mathbf{0 . 2 7 1}$ & 4.167 & $\mathbf{1 . 0 4 4}$ \\
\hline \multicolumn{5}{|c|}{ FLOWER } \\
\hline SAD & 2.537 & 1.143 & 2.768 & 1.407 \\
ZNCC & 2.018 & 1.106 & 2.920 & 1.467 \\
SIFT & 2.795 & 1.183 & 4.747 & 2.480 \\
LIFT & 3.049 & 1.420 & 4.224 & 2.358 \\
OUR concat & 2.033 & 0.843 & $\mathbf{2 . 6 0 9}$ & 1.267 \\
OUR & $\mathbf{1 . 9 7 3}$ & $\mathbf{0 . 7 7 1}$ & $\mathbf{2 . 6 0 9}$ & $\mathbf{1 . 2 0 8}$ \\
\hline \multicolumn{5}{|c|}{ CAN } \\
\hline SAD & 1.824 & 0.664 & 2.283 & 1.156 \\
ZNCC & 1.187 & 0.628 & 2.092 & 1.098 \\
SIFT & 1.769 & 0.874 & 3.067 & 1.726 \\
LIFT & 2.411 & 1.207 & 3.003 & 1.823 \\
OUR concat & $\mathbf{1 . 0 8 2}$ & $\mathbf{0 . 4 7 7}$ & $\mathbf{1 . 8 9 6}$ & $\mathbf{0 . 8 3 3}$ \\
OUR & 1.123 & 0.478 & 1.982 & 0.874 \\
\hline \multicolumn{5}{|c|}{ BUDDHA } \\
\hline SAD & 0.849 & 0.250 & 1.119 & 0.561 \\
ZNCC & 0.688 & 0.299 & 1.208 & 0.656 \\
SIFT & 0.696 & 0.263 & 1.347 & 0.618 \\
LIFT & 0.688 & 0.299 & 1.208 & 0.656 \\
OUR concat & 0.682 & 0.231 & $\mathbf{1 . 0 1 7}$ & $\mathbf{0 . 4 7 3}$ \\
OUR & $\mathbf{0 . 6 3 7}$ & $\mathbf{0 . 2 0 6}$ & 1.057 & 0.475 \\
\hline
\end{tabular}

Table 2. Quantitative results for BIRD, FLOWER, CAN, and BUDDHA objects. Six similarity measures are compared: sum of absolute differences, zero mean normal cross correlation, SIFT, LIFT, proposed multi-view similarity using concatenation, and proposed multi-view similarity using averaging.

and mean errors, apparently all measures show quite good performance on the rather diffuse surface, whereas outliers due to ambiguous texture inflate the mean values.

Overall, the proposed multi-view patch similarity exhibits the quantitatively and qualitatively best performance. In particular, the experiments support our claim that learning end-to-end multi-view similarity is preferable to comparing learned per-patch descriptors with conventional Euclidean distance, and to a consensus over learned 2-view similarities.

Varying the number of input patches. We go on to demonstrate that the network architecture, in which branches are averaged, can be applied to different numbers of input views without retraining. We run experiments with either three or nine views, respectively patches. Both give reasonable depth estimates (Fig. 5). As expected, the results with only three views are a bit worse. However, using 


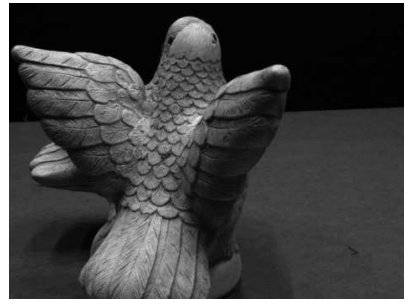

(a) input view

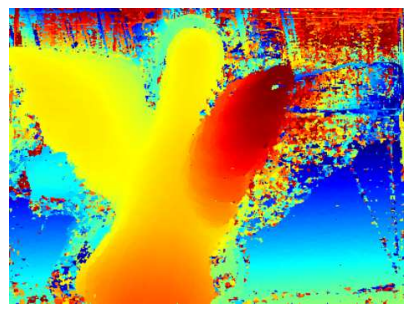

(c) 5 views

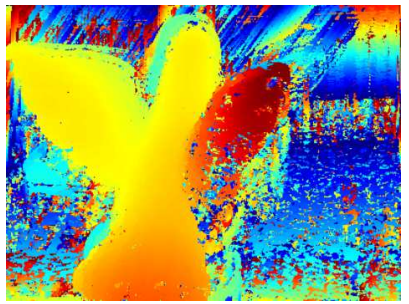

(b) 3 views

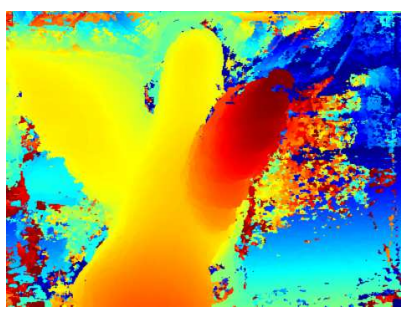

(d) 9 views
Figure 5. Matching different numbers of views with the similarity network can be done without retraining. Result displayed without sub-pixel refinement and box filtering to accentuate differences.

nine patches instead of five further improves performance - although the similarity network has only been trained with five. We speculate that information how to correctly weight pixels at discontinuities, occlusions etc. passes down the individual branches during training, so that the parallel Siamese streams can also use it for additional branches. On the other hand, averaging itself may have a stronger denoising effect with more branches. Further research is needed to clarify the underlying mechanisms. Whatever the reason, in our view flexibility with respect to the number of input views is an attractive and important feature for real-world applications of multi-view stereo.

\subsection{Evaluation on the Fountain dataset}

The images recorded with the robot for the DTU dataset are all taken in an indoor laboratory environment, and one would expect that the specific lighting and camera characteristics of the dataset are captured in our trained network. Therefore, we apply the learned multi-view similarity measure also to the well-known Fountain dataset [22], without retraining it.

For the experiment we select five neighboring images and set the depth range to $[5 \ldots 10] \mathrm{m}$. The depth maps in Fig. 6 show the qualitative result for three different methods. Our method works at least as well as ZNCC and SIFT, which are generic and not learned from data. The network does not seem to significantly overfit to the DTU setting.

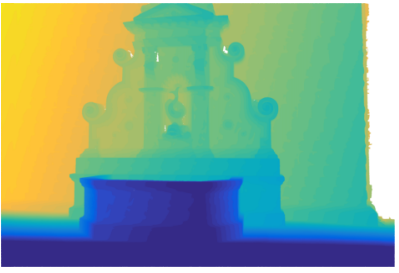

(a) ground truth

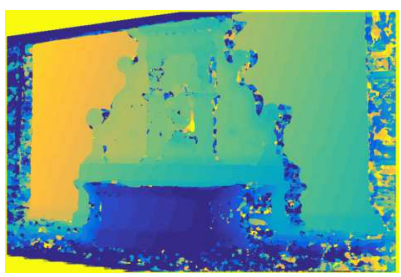

(c) SIFT

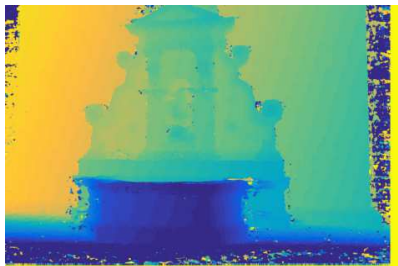

(b) OUR

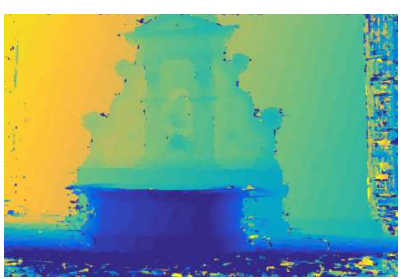

(d) ZNCC
Figure 6. The learned similarity generalizes to a different test environment, seemingly as well as the competing descriptors.

\section{Conclusion}

We have proposed to directly learn a similarity / matching score over a set of multiple patches with a discriminative learning engine, rather than heuristically assemble it from pairwise comparisons. An $n$-way Siamese convolutional network, which applies the same, learned transformation to all input patches and combines the results, was identified as a suitable state-of-the-art learning engine.

From a high-level perspective, machine learning for $3 \mathrm{D}$ vision started with very small steps like learning the distance metric between given descriptors, or learning descriptor extraction for pairwise matching. Recently, bolder ideas have emerged, all the way to learning an end-to-end mapping from images to (volumetric, low-resolution) 3D models [3]. Our work is positioned somewhere in the middle. While we do not see an immediate reason to replace the geometrically transparent and well-understood structure-frommotion pipeline with a learned black-box predictor, we do agree that certain steps of the pipeline lack a principled solution and might be best addressed with machine learning. In our work, we have used the learned similarity score for multi-view stereo matching, but we believe that variants of it could also be beneficial for other applications where similarity, respectively distance, between more than two images must be assessed.

Dedication. We dedicate this paper to the memory of Wilfried Hartmann, who did not live to see it published.

Acknowledgements. The work was funded in part by the EU's Horizon 2020 programme under grant agreement 687757 - REPLICATE, and supported by NVIDIA Corporation through an Academic Hardware Grant. We thank D. Marmanis and K.K. Maninis for valuable comments. 


\section{References}

[1] S. T. Barnard and M. A. Fischler. Computational stereo. ACM Computing Surveys, 14(4):553-572, 1982.

[2] C. Barnes, E. Shechtman, A. Finkelstein, and D. Goldman. PatchMatch: a randomized correspondence algorithm for structural image editing. ACM Transactions on Graphics, 28(3), 2009.

[3] C. B. Choy, D. Xu, J. Gwak, K. Chen, and S. Savarese. 3DR2N2: A unified approach for single and multi-view 3d object reconstruction. ECCV 2016.

[4] R. T. Collins. A space-sweep approach to true multi-image matching. In CVPR 1996.

[5] A. Delaunoy, E. Prados, P. G. I. Piracés, J.-P. Pons, and P. Sturm. Minimizing the multi-view stereo reprojection error for triangular surface meshes. BMVC 2008.

[6] S. Galliani, K. Lasinger, and K. Schindler. Massively parallel multiview stereopsis by surface normal diffusion. In ICCV 2015.

[7] S. Galliani and K. Schindler. Just look at the image: viewpoint-specific surface normal prediction for improved multi-view reconstruction. In CVPR 2016.

[8] A. Geiger, P. Lenz, and R. Urtasun. Are we ready for autonomous driving? the KITTI vision benchmark suite. In CVPR 2012.

[9] X. Han, T. Leung, Y. Jia, R. Sukthankar, and A. C. Berg. MatchNet: Unifying feature and metric learning for patchbased matching. In CVPR 2015.

[10] C. Häne, L. Heng, G. H. Lee, A. Sizov, and M. Pollefeys. Real-time direct dense matching on fisheye images using plane-sweeping stereo. In 3DV 2014.

[11] M. J. Hannah. Computer Matching of Areas in Stereo Images. $\mathrm{PhD}$ thesis, Stanford University, 1974.

[12] R. Jensen, A. Dahl, G. Vogiatzis, E. Tola, and H. Aans. Large scale multi-view stereopsis evaluation. In CVPR 2014.

[13] Y. Jia, E. Shelhamer, J. Donahue, S. Karayev, J. Long, R. Girshick, S. Guadarrama, and T. Darrell. Caffe: Convolutional architecture for fast feature embedding. arXiv preprint arXiv:1408.5093, 2014.

[14] T. Kanade, A. Yoshida, K. Oda, H. Kano, and M. Tanaka. A stereo machine for video-rate dense depth mapping and its new applications. In CVPR 1996.

[15] S. Katz, A. Tal, and R. Basri. Direct visibility of point sets. In ACM SIGGRAPH 2007.

[16] D. Lowe. Distinctive image features from scale-invariant keypoints. IJCV, 60(2):91-110, 2004.

[17] D. Scharstein and R. Szeliski. A taxonomy and evaluation of dense two-frame stereo correspondence algorithms. International Journal of Computer Vision, 47(1-3):7-42, 2002.

[18] T. Schöps, J. Schnberger, S. Galliani, T. Sattler, K. Schindler, M. Pollefeys, and A. Geiger. A multi-view stereo benchmark with high-resolution images and multi-camera videos. In CVPR 2017.

[19] S. M. Seitz, B. Curless, J. Diebel, D. Scharstein, and R. Szeliski. A comparison and evaluation of multi-view stereo reconstruction algorithms. In CVPR 2006.
[20] E. Simo-Serra, E. Trulls, L. Ferraz, I. Kokkinos, P. Fua, and F. Moreno-Noguer. Discriminative learning of deep convolutional feature point descriptors. In ICCV 2015.

[21] K. Simonyan, A. Vedaldi, and A. Zisserman. Learning local feature descriptors using convex optimisation. IEEE Transactions on Pattern Analysis and Machine Intelligence, 36(8):1573-1585, Aug 2014.

[22] C. Strecha, W. V. Hansen, L. J. V. Gool, P. Fua, and U. Thoennessen. On benchmarking camera calibration and multi-view stereo for high resolution imagery. CVPR 2008.

[23] K. M. Yi, E. Trulls, V. Lepetit, and P. Fua. LIFT: learned invariant feature transform. CoRR, abs/1603.09114, 2016.

[24] J. Yu, Q. Tian, J. Amores, and N. Sebe. Toward robust distance metric analysis for similarity estimation. In $C V P R$ 2006.

[25] C. Zach. Fast and high quality fusion of depth maps. In 3DPVT 2008.

[26] S. Zagoruyko and N. Komodakis. Learning to compare image patches via convolutional neural networks. In $C V P R$ 2015.

[27] J. Zbontar and Y. LeCun. Computing the stereo matching cost with a convolutional neural network. In CVPR 2015. 